\title{
Broadband terahertz generation with a stair-step echelon
}

\author{
Koustuban Ravi ${ }^{1,2}$, Benjamin K.Ofori-Okai ${ }^{3}$, Prasahnt Sivarajah ${ }^{3}$, Wenqian Ronny Huang ${ }^{2}$, Franz X. \\ Kärtner $^{1,2,4}$, and Keith A.Nelson ${ }^{3}$ \\ 1. Center for Free-Electron laser science, DESY, Notkestraße 85, Hamburg 22607, Germany \\ 2. Massachusetts Institute of Technology, Department of Electrical Engineering and Computer Science \\ 3. Massachusetts Institute of Technology, Department of Chemistry \\ 4. Department of Physics, University of Hamburg, Hamburg 22761, Germany. \\ koust@mit.edu
}

\begin{abstract}
A method to overcome limitations of conventional broadband terahertz generation techniques is presented. A stair-step echelon allows for the creation of superior tilted-pulse-fronts to yield larger frequencies and bandwidths, energy conversion efficiencies exceeding $5 \%$.

OCIS codes: (190.0190) Nonlinear optics; (300.6495) Terahertz spectroscopy
\end{abstract}

\section{Introduction}

Ultrafast terahertz (THz) transients are of great interest for linear and nonlinear $\mathrm{THz}$ spectroscopy and compact particle acceleration [1]. Tilted-pulse-front (TPF) [2] THz generation in lithium niobate (LN) has become ubiquitous due to its compatibility with easily accessible high-power $800 \mathrm{~nm} / 1 \mu \mathrm{m}$ laser technology. However, the use of diffraction grating (DG) based TPFs (DG-TPFs) present formidable imaging challenges, particularly for large pump bandwidths and beam sizes. Furthermore, as the optical pump spectrum red-shifts and broadens due to repeated down-conversion to $\mathrm{THz}$ frequencies (referred to as cascading), a spatio-temporal break-up of the pump pulse results [3]. This limits the energy conversion efficiency and produces THz pulses with spatiotemporal distortions. In order to address this issue, terahertz generation in organic materials and semiconductors have been proposed and demonstrated [4-5]. However, they rely on the development of pump lasers at wavelengths $>1.3 \mu \mathrm{m}$, which maybe more challenging in relation to well-developed $1 \mu \mathrm{m}$ laser technology. We describe new mechanisms to eliminate these limitations by engineering the transverse-momentum distribution of the pump pulse via a stair-step echelon [6] to produce TPFs which do not contain the same distortions as DGTPFs. This constitutes one of two approaches [7] presented by us towards scaling single-cycle pulse energies from the current $\mathrm{mJ}$ level to the $10 \mathrm{~mJ}$ level.

\section{Results}

The structure (Fig.1a) produces a discrete optical pulse front composed of many beamlets. Each beamlet propagating in $\mathrm{LN}$ at the optical group velocity $c / n_{\mathrm{g}}$ produces a $\mathrm{THz}$ wavelet that propagates at phase velocity $c / n_{\mathrm{THz}}$ in a direction given by the Cherenkov angle $\gamma=\cos ^{-1}\left(n_{\mathrm{THz}} / n_{\mathrm{g})}\right.$ where $\gamma=63^{\circ}$ for LN. The THz wavelets superpose constructively if the time delay $\Delta t$ and spatial offset $\Delta x$ between successive optical beamlets are related by $\Delta t=\Delta x \tan \gamma n_{\mathrm{g}} / c$ (Fig.1c), yielding a single-cycle THz plane wave with uniform spatial properties (Fig.1d).
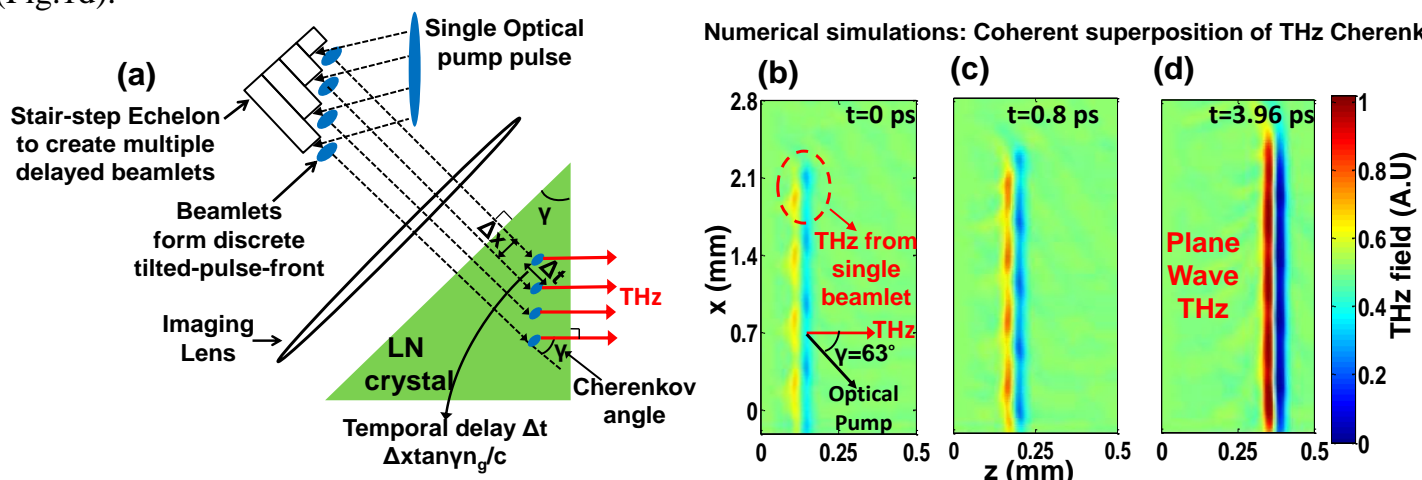

Fig.1(a) Schematic illustration of echelon depicting THz generation by relatively delayed optical beamlets. (b)-(d) Numerical simulations of echelon : Individual $\mathrm{THz}$ wavelets superpose coherently when the temporal delay $\Delta \mathrm{t}$ and spatial offset $\Delta \mathrm{x}$ between beamlets are related by $\Delta \mathrm{t}=\Delta \mathrm{xtan} \gamma \mathrm{n}_{\mathrm{g}} / \mathrm{c}$, where $\gamma=63^{\circ}$ is the Cherenkov angle for $\mathrm{LN}$ 
(a)

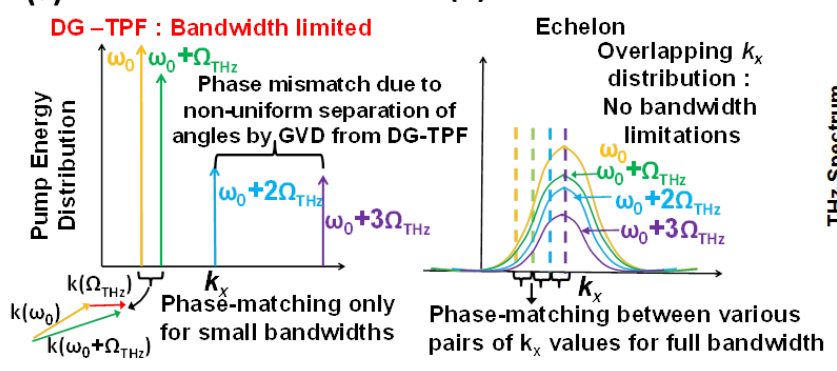

(c)

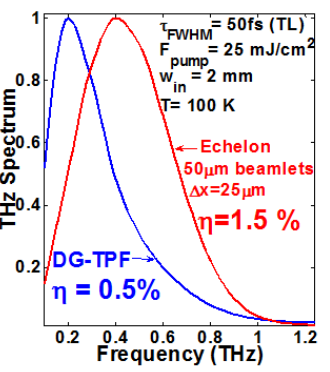

(d)

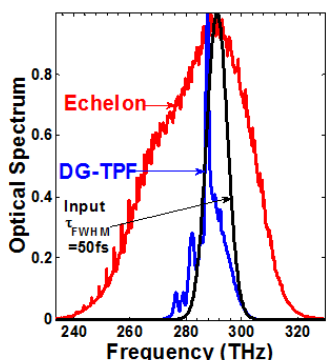

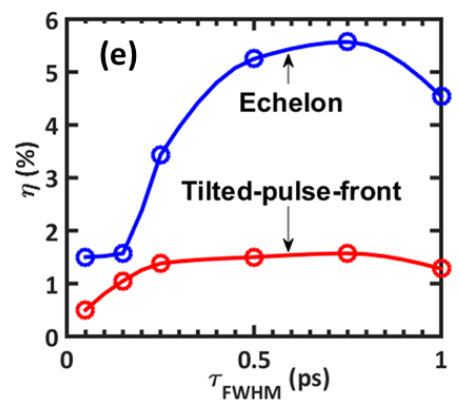

Fig.2.(a) Inherent bandwidth limitation due to group velocity dispersion(GVD) produced by DG- TPFs. Phase-matching conditions are satisfied only for small bandwidths. (b) Echelons contain overlapping transverse momentum $\left(\mathrm{k}_{\mathrm{x}}\right)$ distributions, which solves the bandwidth limitation. (c) Higher conversion efficiencies, broader $\mathrm{THz}$ spectra predicted for echelons. (d) Spectral broadening due to cascading in echelons is larger than with DG-TPFs.(e) Echelons produce conversion efficiencies much larger than conventional TPFs.

In DG-TPFs, each frequency component of the pump is mapped to a different angle (or transverse momentum $k_{x}$ ) due to angular dispersion (Fig.2a). However the variation of angle with frequency is non-uniform due to group velocity dispersion caused by angular dispersion. Consequently, phase-mismatch occurs for large pump bandwidths or due to cascading (Fig.2a). For the echelon, when beamlets are sufficiently sparse, all frequencies have an overlapping distribution in $k_{x}(2 \mathrm{~b})$, which circumvents bandwidth limitations. In Fig.2c, THz spectra resulting from DG-TPFs and echelons are compared theoretically. The simulations incorporate spatio-temporal distortions, cascading, absorption and dispersion [7]. For the conditions in Fig.2c, significantly larger conversion efficiencies with broader spectra and larger $\mathrm{THz}$ frequencies are observed for the echelon. This is consistent with trends from experiments [3]. The pump spectral broadening due to cascading is also larger (Fig.2d).

\section{Conclusion}

The use of echelons for $\mathrm{THz}$ generation may result in significantly higher conversion efficiencies of several percent $(\eta=5 \%$ for 0.5 ps pump pulses is predicted for echelons (Fig. 2e), superior beam properties, and broader $\mathrm{THz}$ spectra by eliminating limitations posed by DG-TPFs. The method also alleviates some experimental challenges, particularly for large beam sizes. In comparison to our proposed aperiodically poled structures [7], the advantage is the ease of availability of large aperture crystals while the disadvantage is the relatively lesser flexibility in terahertz pulse shaping.

\section{References}

[1] W.R.Huang, A.Fallahi, X.Wu, H.Cankaya, A.L.Calendron, K.Ravi, D.Zhang, E.A.Nanni, K.H.Hong, and F.X.Kärtner, "Terahertzdriven all-optical electron gun, " Optica 3,1209-1212 (2016).

[2] J. Hebling et al, Opt. Express, 10 (21), 1161 (2002).

[3] K.Ravi et al., "Theory of terahertz generation by optical rectification using tilted-pulse-fronts", Opt. Express 23,5253 (2015).

[4] C. Vicario, A. Ovchinnikov, S. Ashitkov, M. Agranat, V. Fortov, and C. Hauri, Optics letters 39, 6632 (2014).

[5] J. Fülöp, G. Polónyi, B. Monoszlai, G. Andriukaitis, T. Balciunas, A. Pugzlys, G. Arthur, A. Baltuska, and J. Hebling, Optica 3, 1075 (2016).

[6] B.K. Ofori-Okai, P. Sivarajah, Wenqian Ronny Huang and Keith A.Nelson, "THz generation using a reflective stair-step echelon,” Opt. Express 24, 5057-5068 (2016)

[7] K.Ravi, A.Yahaghi, A.Fallahi, and F.X.Kärtner, "Aperiodically poled structures for high efficiency broadband terahertz generation," submitted CLEO'17. 